\title{
УДОВЛЕТВОРЕНИЕ БАЗОВЫХ ПСИХОЛОГИЧЕСКИХ ПОТРЕБНОСТЕЙ КАК ИСТОЧНИК ТРУДОВОЙ МОТИВАЦИИ И СУБЪЕКТИВНОГО БЛАГОПОЛУЧИЯ У РОССИЙСКИХ СОТРУДНИКОВ
}

\author{
Е.Н. ОСИН, Д.Д. СУЧКОВ, Т.О. ГОРДЕЕВА, Т.Ю. ИВАНОВА
}

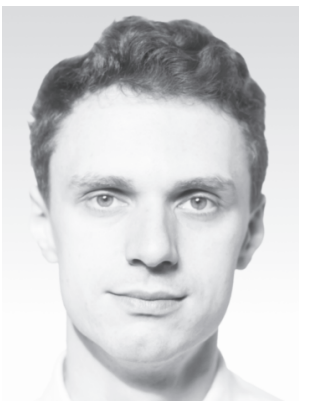

Осин Евгений Николаевич - ведущий научный сотрудник международной лаборатории позитивной психологии личности и мотивации НИУ ВШЭ, доцент департамента психологии НИУ ВШЭ, кандидат психологических наук.

Контакты: evgeny.n.osin@gmail.com

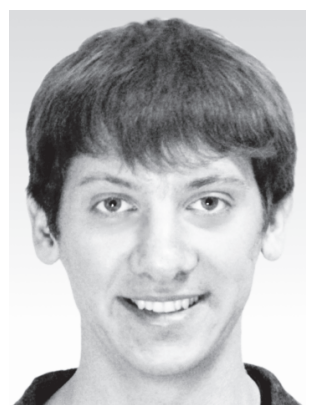

Сучков Дмитрий Дмитриевич - младший научный сотрудник международной лаборатории позитивной психологии личности и мотивации НИУ ВШЭ, аспирант департамента психологии НИУ ВШЭ. Контакты: dsuchkov@hse.ru

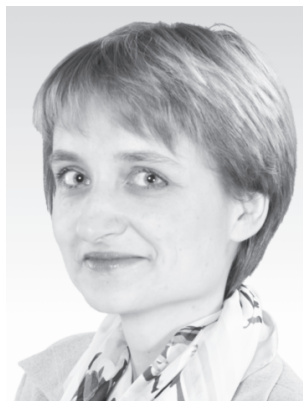

Гордеева Тамара Олеговна - доцент факультета психологии МГУ имени М.В. Ломоносова, ведущий научный сотрудник международной лаборатории позитивной психологии личности и мотивации НИУ ВШЭ, доктор психологических наук.

Контакты: tamgordeeva@gmail.com 


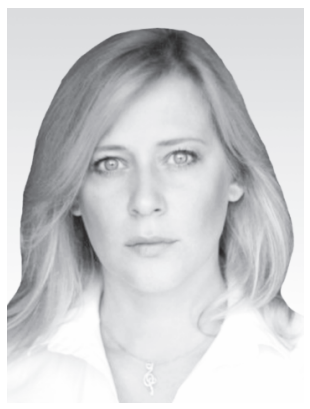

Иванова Татьяна Юрьевна - научный сотрудник международной лаборатории позитивной психологии личности и мотивации НИУ ВШЭ.

Контакты: tatiana.y.ivanova@gmail.com

\section{Резюме}

Удовлетворение базовых психологических потребностей в автономии, компетентности и связанности с другими людьми, постулируемое в теории самодетерминации Э. Диси и Р. Райана, рассматривается как важный предиктор оптимального функционирования индивидов. В статье представлены результаты русскоязычной адаптации Шкалы удовлетворенности базовых психологических потребностей на работе, предложенной Э. Диси с коллегами. Использована выборка сотрудников крупной производственной организации $(\mathrm{N}=1183)$. С использованием структурного моделирования показано, что структура опросника соответствует теоретически ожидаемой бифакторной модели, включающей три фактора базовых потребностей и два фактора систематической ошибки, связанной с направлением утверждений. Разработанные шкалы обладают приемлемой внутренней согласованностью ( $\alpha$ Кронбаха 0.70-0.85). Для проверки конструктной валидности шкал использованы опросник трудовой мотивации, опирающийся на теорию самодетерминации, а также индикаторы субъективного благополучия - шкалы удовлетворенности жизнью и позитивного и негативного аффекта. Шкалы трех базовых потребностей демонстрируют слабые и умеренные позитивные связи с показателями удовлетворенности жизнью и позитивного аффекта, а также внутренней, интегрированной, идентифицированной и интроецированной мотивации; негативные связи - с показателями негативного аффекта, экстернальной мотивации и амотивации. С использованием структурного моделирования показано, что автономная и контролируемая трудовая мотивация может рассматриваться как медиатор связей удовлетворения базовых потребностей с субъективным благополучием. Полученные результаты соответствуют положениям теории самодетерминации и данным зарубежных исследований и свидетельствуют о важности разработки интервенций, направленных на повышение удовлетворенности базовых потребностей сотрудников организаций. Обсуждаются основные принципы разработки таких интервенций с опорой на теорию самодетерминации.

Ключевые слова: базовые потребности, Шкала удовлетворенности базовых потребностей, теория самодетерминации, потребность в автономии, потребность в компетентности, потребность в связанности с другими, внутренняя трудовая мотивация, автономная мотивация, психологическое благополучие.

\section{Теоретические основы исследования}

Многие теоретики мотивации рассматривают потребности как фун- даментальные побудители человеческого поведения. Например, А. Маслоу предлагает пять иерархических уровней потребностей, ранжируемых от физиологических нужд до 
самоактуализации. Д. Макклеланд утверждает, что люди различаются по степени выраженности у них различных потребностей, акцентируя важность таких потребностей, как потребности в достижении, в аффилиации и власти. В теории самодетерминации (СДТ) Э. Диси и Р. Райана выделяются три врожденные базовые потребности (БП) - в автономии, компетентности и связанности, удовлетворение которых рассматривается как условие возникновения внутренней мотивации и в целом достижения оптимального функционирования (Ryan, Deci, 2000). Напротив, фрустрация БП подрывает счастье, рост и здоровое развитие индивидов и ведет к неблагополучию. Первые эмпирические подтверждения данных положений начали появляться в конце 1990-х гг. Количество исследований в русле СДТ в последние годы постоянно растет, демонстрируя благоприятные последствия удовлетворения базовых потребностей (эти исследования подробно представлены на сайте теории: http://www.selfdeterminationtheory.org). В последние десятилетия СДТ является лидирующей среди теорий мотивации, а Э. Диси и Р. Райан входят в число наиболее цитируемых авторов в психологии и психиатрии.

Базовые психологические потребности - центральный конструкт теории самодетерминации (Deci, Ryan, 2000). Идея о трех базовых потребностях (БП) лежит в основе понимания феноменов внутренней и внешней мотивации, оптимального функционирования личности, ее психологического благополучия и творческих достижений. Именно эти три потребности так или иначе лежат в основе всех мини-теорий, составляющих СДТ. Особое значение придается потребности в автономии, исследованию эффектов ее поддержки, а также разработке практических рекомендаций по обеспечению ее удовлетворения. Несмотря на то что у человека может быть выделено Значительно большее число потребностей (Sheldon et al., 2001), только три отвечают критерию потребностей, предложенному авторами СДТ, как необходимых условий психологического благополучия и роста (потребности, не удовлетворяющие этому критерию, в СДТ предлагается называть желаниями). Как вода и солнце нужны растению, чтобы жить и развиваться, так и у человека вне зависимости от его возраста, пола и культурной принадлежности должны быть удовлетворены его БП; Р. Райан называет три БП «психологической пищей» (Ryan, 1995), необходимой для процветания и роста индивида. От рождения они изначально заданы у всех человеческих существ и не требуют формирования, но нуждаются в постоянной поддержке со стороны окружения родителей, учителей, работодателей, близких, общества в целом.

Потребность в автономии означает стремление самостоятельно контролировать собственные действия и поведение, быть их независимым инициатором, стремление чувствовать выбор и собственную детерминацию своего поведения. Это универсальная потребность ощущать себя субъектом, источником предпринимаемой активности, ее независимым инициатором, причиной собственных действий, стремление 
действовать в гармонии со своим интегрированным Я, а не быть объектом воздействия других людей, их влияния и контроля. При этом чувствовать автономию своего поведения, своей жизни не значит быть независимым от других.

Под потребностью в компетентности понимается стремление индивида чувствовать себя эффективным деятелем, способным справляться с разного уровня сложности задачами той среды, в которой он находится, причем достигаемые результаты могут быть как внешними, так и внутренними. Важность этой потребности подчеркивалась такими исследователями, как Р. Вудвортс, Р. Уайт, А. Маслоу, С. Хартер; важность поддержки у ребенка чувства компетентности подчеркивал в своей теории развития личности Э. Эриксон. Изначально Диси предположил, что потребность в компетентности заставляет людей стремиться к поиску и решению трудных задач, оптимальных с точки зрения уровня их способностей, что приводит к ощущению мастерства и компетентности. Поскольку состояния самодетерминации и компетентности важны для внутренней мотивации и очень тесно связаны между собой, Диси и Райан вводят конструкт самодетерминированной компетентности.

Наконец, третья базовая потребность - во взаимосвязи с другими людьми или связанности (relatedness) - касается качества человеческих отношений, она включает стремление к установлению надежных и удовлетворяющих индивида отношений с другими людьми, основанных на чувстве привязанности и дающих ощущение принятия, поня- тости, поддержки. По сути, она означает желание быть частью группы, любить и заботиться о других людях и получать любовь и заботу с их стороны. Аналоги потребности в связанности можно найти и у других теоретиков мотивации: например, в иерархии потребностей А. Маслоу, теории привязанности Дж. Боулби и М. Эйнсворт, теории Р. Баумайстера и М. Лири.

Поскольку, согласно СДТ, эти потребности являются врожденными, изначально заданными у всех людей, вопрос ставится не о степени индивидуальных различий в выраженности каждой потребности, а о мере ее фрустрации (или удовлетворенности) со стороны окружения индивида. Особое значение в теории придается потребности в автономии, и именно она наиболее активно исследуется авторами концепции и их последователями. Если аналоги потребности компетентности и связанности можно найти и у других теоретиков мотивации, то теории, ставящие во главу угла потребность в автономии, отсутствуют. Хотя некоторые теоретики были близки к этой идее: она высказывалась Р. Де Чармсом; И.П. Павлов и М.М. Убергриц выделили еще в начале ХХ в. «рефлекс свободы», который возникает у живого организма в ответ на ограничение его свободной активности.

Потребность в автономии самая важная, ключевая для функционирования внутренней мотивации. Для поддержания и усиления внутренней мотивации субъект должен переживать свое поведение как самодетерминированное. Потребность в компетентности также важна, однако 
недостаточна для поддержания внутренней мотивации. Удовлетворение третьей потребности - в связанности с другими людьми - является третьим важным условием (источником) развития и поддержания внутренней мотивации.

С точки зрения СДТ удовлетворение всех трех БП также важно для психологического благополучия, роста, успешного становления и функционирования личности (Deci, Ryan, 2000; Reis et al., 2000). Если лишь одна или две БП будут удовлетворены, пусть даже в высокой степени, при неудовлетворении хотя бы одной БП психологическое здоровье индивида будет страдать (Sheldon, Niemiec, 2006). Исследования также показывают, что имеет значение, насколько сбалансированным является удовлетворение БП в основных значимых для индивида областях жизни (Milyavskaya et al., 2009).

В контексте трудовой деятельности два исследования, проведенных в больших банках США, показали, что сотрудники, чувствовавшие бо́льшую поддержку автономии со стороны своих менеджеров, испытывали бо́льшую удовлетворенность БП на работе, демонстрировали более высокий уровень трудовых достижений и более высокие показатели психологического благополучия (операционализированного в терминах витальности, удовлетворенности жизнью, самооценки), а также более низкие показатели психологического неблагополучия (тревожности, депрессии и соматизации) (Baard et al., 2004).

Другие исследования в организационном контексте также показали, что удовлетворение БП позитивно связано с оптимальным функционированием на рабочем месте - удовлетворенностью работой, вовлеченностью в работу, внутренней мотивацией и благополучием на рабочем месте (Kasser, Ryan, 1999) и негативно - со стрессом на рабочем месте, синдромом выгорания, а также негативными аттитюдами в отношении работы, такими как намерение оставить работу (Van den Broeck et al., 2008). В исследованиях, рассматривавших вклад каждой из трех БП по отдельности, было показано, что каждая БП обнаруживает независимую связь с внутренней мотивацией и оптимальным функционированием сотрудников (Lynch et al., 2005).

Обращение к конструкту БП способствовало развитию понимания вклада организационных факторов, объясняя связи между стилем лидерства в организации, характеристиками работы, с одной стороны, и благополучием и достижениями сотрудников, с другой (Deci et al., 2001). Было также показано, что некоторые личностные и организационные переменные связаны с удовлетворением трех БП: например, оптимизм и процедурная справедливость были позитивно связаны с БП, постулируемыми в СДТ (Van den Broeck et al., 2010).

Существующие методики для измерения БП. Учитывая важность конструкта БП, актуальна разработка инструментов для их диагностики. К настоящему времени разработаны шкалы, диагностирующие БП в целом (общие шкалы БП см.: Sheldon, Hilpert, 2012). Также существуют опросники БП по отдельным сферам жизни - на работе, в школе, спорте, межличностных отношениях. 
Поскольку в рамках данной статьи нас интересует диагностика БП на рабочем месте, остановимся более подробно на опросниках этого типа. Группой бельгийских исследователей была разработана сбалансированная шкала БП на работе (см.: Van den Broeck et al., 2010), включающая утверждения, отражающие как удовлетворение БП, так и их фрустрацию. Однако эта шкала была подвергнута критике, согласно которой ряд утверждений шкалы автономии отражает не само ее удовлетворение, а необходимы для этого условия.

Еще один опросник БП на работе был предложен М. Брайен с соавт. (Brien et al., 2012) на французском языке и валидизирован в двух культурах (канадской и французской). Этот опросник включает только позитивные (прямые) утверждения, по четыре на каждую шкалу. Кроме того, ряд утверждений этой методики не вполне соответствует операционализации потребностей, принятой в СДТ. Например, утверждения «Я могу взять на себя ответственность за свою работу» (шкала автономии) может рассматриваться как результат удовлетворения потребности, а не собственно ощущение ее удовлетворения.

Э. Диси с соавт. разработали опросник, оценивающий БП на работе (см.: Deci et al., 2001; Baard et al., 2004). Именно эта шкала представлена на сайте теории самодетерминации как основной инструмент диагностики базовых потребностей на работе и использовалась в целом ряде исследований. Таким образом, целью настоящего исследования стала русскоязычная адаптация Опросника базовых потребностей в трудовой деятельности (The Basic Need Satisfaction at Work Scale) на выборке сотрудников коммерческой организации.

\section{Методика}

Выборка. В исследовании приняли участие сотрудники $(\mathrm{N}=1183)$ производственной организации с филиалами в шести регионах Центрального и Северо-Западного федеральных округов России. Выборка включала 422 женщины и 761 мужчину, в возрасте от 18 до 77 лет ( $\mathrm{M}=$ $=40.0 ; \mathrm{SD}=12.4$ года).

Инструменты. Для диагностики степени удовлетворения базовых психологических потребностей сотрудников организации был подготовлен русский перевод Шкалы удовлетворенности базовых потребностей на работе. Опросник включает 21 утверждение, согласие с каждым из которых оценивается по 7-балльной шкале (от 1 «совершенно не верно» до 7 «совершенно верно»). В каждой шкале используются как прямые, так и обратные утверждения.

Для оценки конструктной валидности опросника применялись Шкала трудовой мотивации (Осин, Иванова, Гордеева, 2013; новая версия готовится к печати). Опросник состоит из 20 утверждений, оцениваемых по 5-балльной шкале и сгруппированных в 6 шкал, измеряющих различные формы мотивации, согласно теории самодетерминации: внутреннюю $(\alpha=0.91)$, внешнюю интегрированную $(\alpha=0.88)$, внешнюю идентифицированную $(\alpha=$ $=0.82)$, внешнюю интроецированную $(\alpha=0.72)$, внешнюю экстерналь- 
ную $(\alpha=0.79)$ и амотивацию $(\alpha=$ $=0.72)$.

Для диагностики субъективного благополучия использовались: Шкала удовлетворенности жизнью Э. Динера (SWLS) в адаптации Д.А. Леонтьева и Е.Н. Осина (Осин, Леонтьев, 2008) ( $\alpha=0.87)$, сокращенная версия Шкалы позитивного и негативного аффекта (ШПАНА: Осин, 2012) из 12 утверждений $(\alpha=$ $=0.87$ и 0.91 для показателей позитивного и негативного аффекта соответственно).

Прочедура исследования. Опросники предъявлялись сотрудникам дистанционно, в рамках компьютеризованной батареи методик. Специалисты по кадрам приглашали сотрудников пройти анонимное анкетирование на специально организованных рабочих местах с целью изучения психологического климата в организации.

Статистическая обработка данных проводилась с помощью программ SPSS 20 и Mplus 7.3. Использовались методы сравнения выборок, корреляционный анализ, конфирматорный факторный анализ и путевой анализ.

\section{Результаты и обсуждение}

Факторная структура и надежность методики. Соответствие эмпирической структуры методики теоретической модели проверялось при помощи конфирматорного факторного анализа в системе Mplus 7.3 с использованием робастных статистик (устойчивых по отношению к отклонению распределения данных от нормального вида: метод MLM). Метрика латентных переменных (факторов) задавалась через их дисперсии, которые приравнивались к 1. Для оценки пригодности моделей использовались принятые критерии $(\mathrm{CFI} \geqslant 0.95$, RMSEA $\leqslant 0.05-$ см.: Brown, 2006).

Первой проверялась теоретическая модель 1, в которой были заданы три латентных фактора, отражающих удовлетворенность базовых потребностей в автономии, компетентности и связанности $(7,6$ и 8 утверждений соответственно по ключу для оригинальной методики). Модель 2 представляла собой альтернативную модель с двумя латентными факторами, отражавшими удовлетворенность и неудовлетворенность базовых потребностей (и включавшими 12 прямых и 9 обратных утверждений соответственно). Модель 3 имела бифакторную структуру (см., например: Chen et al., 2012) и включала три свободно коррелирующих фактора удовлетворенности базовых психологических потребностей в автономии, компетентности и связанности, а также два не связанных с ними и друг с другом фактора, отражающих систематическую ошибку, связанную с прямым и обратным направлением утверждений (method factors).

Показатели соответствия моделей данным представлены в таблице 1. Неудовлетворительное соответствие данным модели 1 объясняется наличием прямых и обратных утверждений. Вклад направления утверждений является существенным, однако сам по себе недостаточным для удовлетворительного объяснения полученных данных, о чем свидетельствуют невысокие показатели модели 2. Наилучшее соответствие данным 
Таблища 1

Показатели соответствия альтернативных моделей

\begin{tabular}{|l|c|c|c|c|}
\hline Модель & df & $\chi^{2}, \boldsymbol{p}$ & CFI & RMSEA (90\% CI) \\
\hline 1 (3 фактора, 21 утверждение) & 186 & $1799.63, p<0.001$ & 0.779 & $0.086(0.082,0.089)$ \\
\hline 2 (2 фактора, 21 утверждение) & 174 & $901.53, p<0.001$ & 0.900 & $0.059(0.056,0.063)$ \\
\hline 3 (5 факторов, 21 утверждение) & 165 & $575.11, p<0.001$ & 0.944 & $0.046(0.042,0.050)$ \\
\hline 4 (5 факторов, 20 утверждений) & 147 & $504.60, p<0.001$ & 0.950 & $0.045(0.041,0.050)$ \\
\hline
\end{tabular}

Примечание: df - число степеней свободы, CFI - сравнительный индекс соответствия, RMSEA - корень среднеквадратической ошибки аппроксимации с 90\%-м доверительным интервалом.

показала модель 3, факторы которой отражают как содержание, так и направление утверждений.

В модели 3 все утверждения демонстрировали значимые факторные нагрузки, за исключением утверждения 14, показавшего статистически недостоверную нагрузку на фактор компетентности $(\lambda=-0.03, p>0.1)$. Можно предположить, что утверждение 14 не сработало по причине сложности формулировки («На работе у меня мало возможностей показать, на что я действительно способен(на)»). Ряд авторов также критикуют данный пункт опросника БП, утверждая, что он многозначен (Sheldon, Hilpert, 2012). В итоговой версии опросника это утверждение скорректировано. По итогам анализа индексов модификации было принято решение исключить утверждение 14 из модели. Показатели полученной модели 4 для 20 утверждений свидетельствуют об отличном соответствии данным, а все факторные нагрузки являются статистически достоверными $(p<0.001)$. Модель 4 представлена на рисунке 1.
Показатель надежности $(\alpha)$ составил 0.85 для шкалы связанности, 0.75 для шкалы автономии и 0.70 для шкалы компетентности. Распределения по всем трем шкалам были весьма близки к нормальному виду (значения асимметрии лежали в диапазоне от 0.00 до 0.12 , эксцесса - от -0.66 до 0.03). Коэффициенты корреляции Пирсона шкалы автономии со шкалами компетентности и связанности составили 0.67 и 0.70 , а компетентности и связанности друг с другом -0.72 .

Конструктная валидность метоӘики. Для изучения конструктной валидности мы проанализировали связи шкал удовлетворенности базовых потребностей с показателями трудовой мотивации и субъективного благополучия. Результаты корреляционного анализа представлены в таблице 2.

Удовлетворенность базовых потребностей значимо положительно коррелирует с автономными типами мотивации, причем коэффициенты корреляции снижаются по мере движения по мотивационному континууму в 
Рисунок 1

Факторная модель Шкалы удовлетворенности базовых потребностей на работе $(\mathrm{N}=1183)$

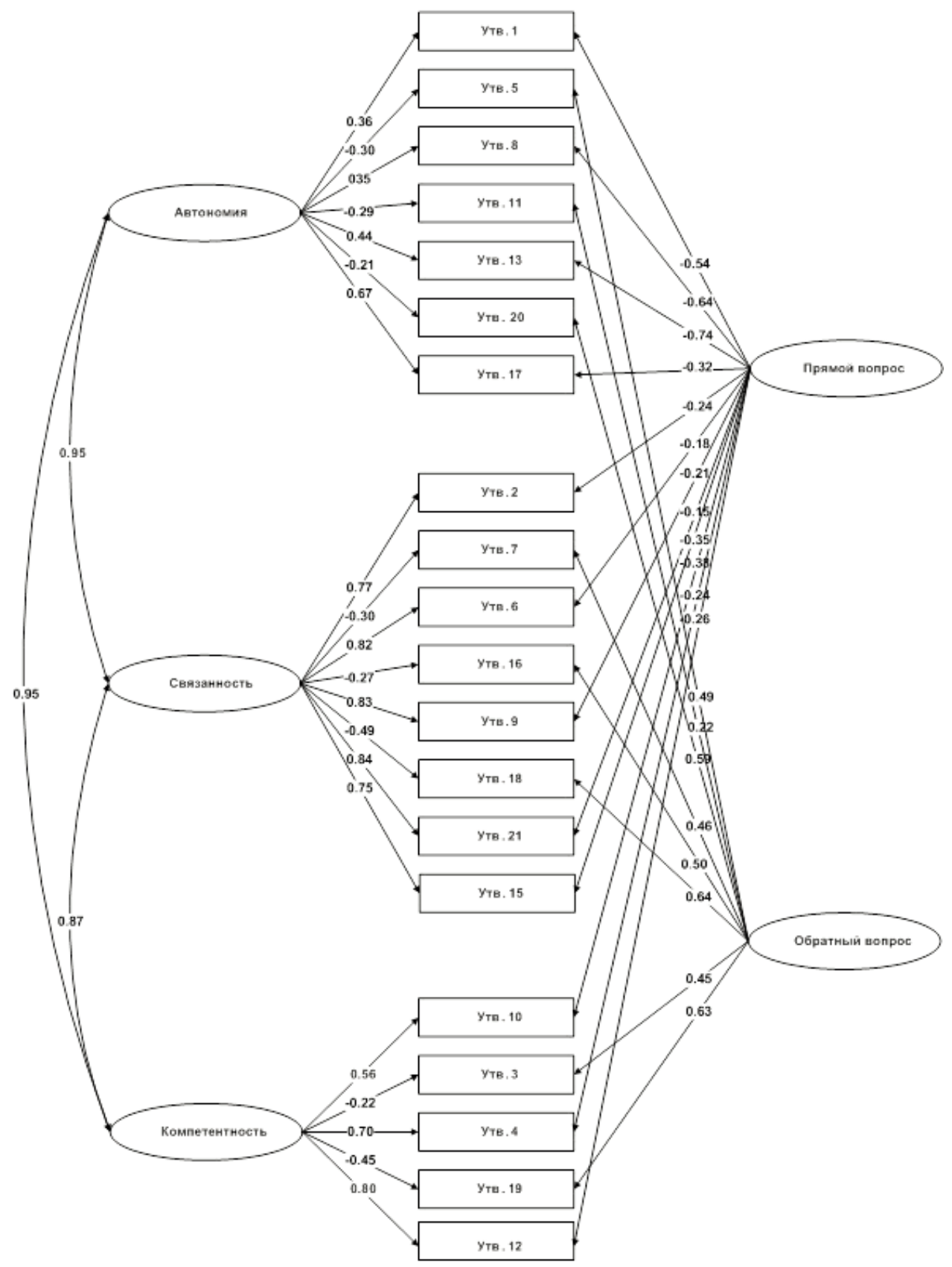


Таблица 2

Коэффициенты корреляции Пирсона шкал удовлетворенности базовых потребностей с показателями трудовой мотивации и субъективного благополучия $(\mathrm{N}=1183)$

\begin{tabular}{|l|l|c|c|c|}
\hline \multicolumn{2}{|c|}{ Шкала } & Автономия & Компетентность & Связанность \\
\hline \multirow{4}{*}{$\begin{array}{l}\text { Трудовая } \\
\text { мотивация }\end{array}$} & Внутренняя & $0.52^{* * *}$ & $0.46^{* * *}$ & $0.42^{* * *}$ \\
\cline { 2 - 5 } & Интегрированная & $0.50^{* * *}$ & $0.42^{* * *}$ & $0.37^{* * *}$ \\
\cline { 2 - 5 } & Идентифицированная & $0.48^{* * *}$ & $0.40^{* * *}$ & $0.34^{* * *}$ \\
\cline { 2 - 5 } & Интроецированная & $0.21^{* * *}$ & $0.11^{* * *}$ & $0.10^{* *}$ \\
\cline { 2 - 5 } & Экстернальная & $-0.18^{* * *}$ & $-0.23^{* * *}$ & $-0.21^{* * *}$ \\
\cline { 2 - 5 } & Амотивация & $-0.27^{* * *}$ & $-0.38^{* * *}$ & $-0.36^{* * *}$ \\
\hline \multirow{4}{*}{\begin{tabular}{l} 
бубъективное \\
\cline { 2 - 5 }
\end{tabular}} & Удовлетв. жизнью & $0.33^{* * *}$ & $0.22^{* * *}$ & $0.25^{* * *}$ \\
\cline { 2 - 5 } & Позитивный аффект & $0.43^{* * *}$ & $0.34^{* * *}$ & $0.36^{* * *}$ \\
\cline { 2 - 5 } & Негативный аффект & $-0.34^{* * *}$ & $-0.26^{* * *}$ & $-0.25^{* * *}$ \\
\hline
\end{tabular}

$* * p<0.01, * * * p<0.001$.

направлении контролируемой мотивации, что соответствует теоретической модели. В соответствии с последней связи шкалы удовлетворенности потребности в автономии с показателями автономной мотивации оказываются более сильными, чем для двух других потребностей. Связи показателей субъективного благополучия с удовлетворенностью базовых потребностей также согласуются с теоретической моделью и данными предыдущих исследований.

С целью проверки положения теории самодетерминации о том, что удовлетворение базовых потребностей поддерживает внутреннюю мотивацию, а та, в свою очередь, вносит позитивный вклад в субъективное благополучие, была построена структурная модель на основе суммарных показателей. Фактор контролируемой мотивации был задан шкалами внешней экстернальной, внешней интроецированной и амотивации. Фактор автономной мотивации был задан шкалами внутренней, внешней интегрированной и внешней идентифицированной мотивации. Показатель интроецированной мотивации имел двойную нагрузку (на фактор как автономной, так и контролируемой мотивации), что соответствует промежуточному положению этой шкалы на мотивационном континууме. Фактор субъективного благополучия был задан в соответствии с теоретическим определением Э. Динера как комбинация удовлетворенности жизнью, негативного аффекта и позитивного аффекта.

Полученная модель продемонстрировала хорошее соответствие данным ( $\mathrm{S}-\mathrm{B} \chi^{2}=258.76$; $\mathrm{df}=45$, $p<0.001$; $\mathrm{CFI}=0.950$; $\mathrm{RMSEA}=$ $=0.063,90 \%$-й доверительный интервал: 0.056-0.071). Статистически 
достоверные $(p<0.05)$ параметры модели представлены на рисунке 2. Согласно этим данным, все три базовые потребности демонстрируют статистически достоверный независимый вклад в показатель автономной мотивации. При этом показатель контролируемой мотивации предсказывает только неудовлетворенность потребности в позитивных отношениях: вероятно, именно при неблагоприятных отношениях в коллективе сотрудники наиболее склонны воспринимать трудовую деятельность как вынужденную. Удовлетворенность базовых потребностей гораздо лучше предсказывала показатель автономной мотивации $\left(R^{2}=\right.$ $=0.33)$, чем показатель контролируемой мотивации $\left(R^{2}=0.04\right)$. Это может быть объяснено тем, что контролируемая мотивация скорее опре- деляется внешними, социальными условиями, в силу которых человек вынужден работать ради денег или не имеет других вариантов работы.

Показатели мотивации хорошо предсказывали субъективное благополучие сотрудников $\left(R^{2}=0.62\right)$ и выступали полными медиаторами связей удовлетворенности базовых потребностей с показателями благополучия. Все непрямые эффекты базовых потребностей на благополучие были статистически достоверными $(p<0.01)$. С учетом высоких корреляций между показателями удовлетворенности трех базовых потребностей была проверена также альтернативная модель, в которой три базовых потребности задавали один латентный фактор. Показатели соответствия этой модели оказались более слабыми $\left(\mathrm{S}-\mathrm{B} \chi^{2}=341.40\right.$;

Рисунок 2

Структурная модель связи удовлетворенности базовых потребностей и трудовой мотивации с субъективным благополучием ( $\mathrm{N}=1183)$

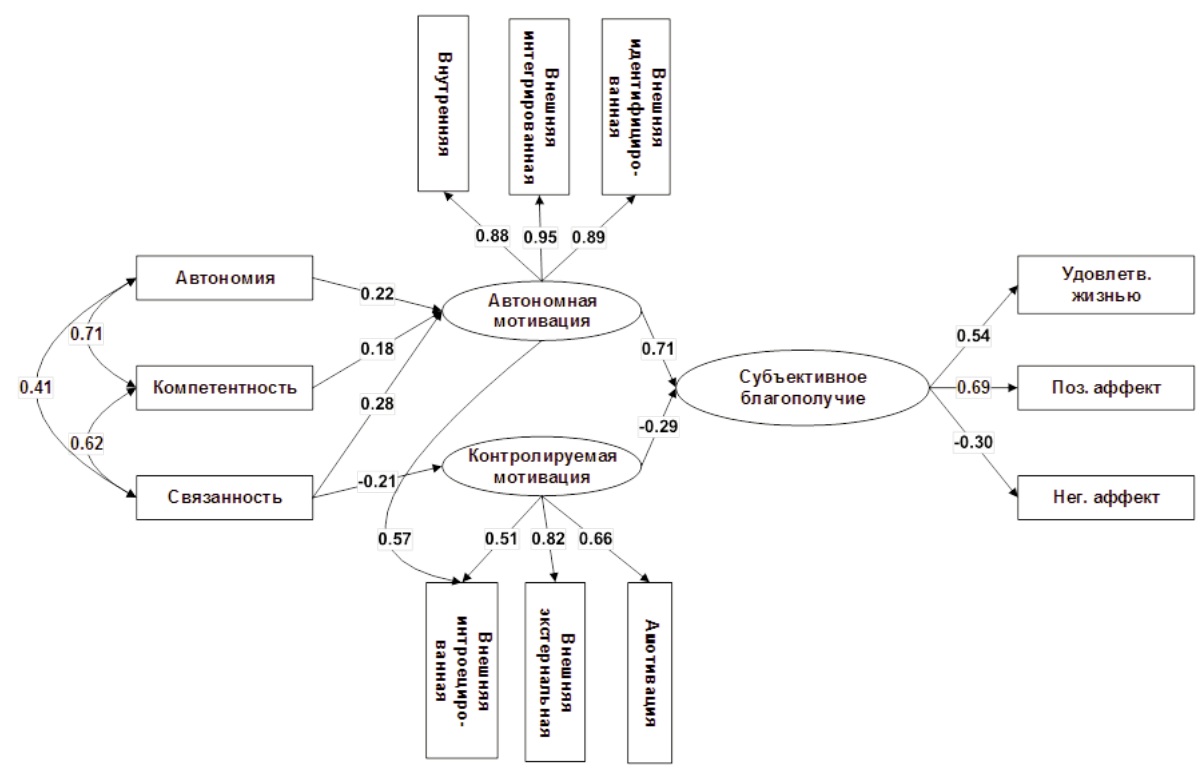


$\mathrm{df}=49, \quad p<0.001 ; \mathrm{CFI}=0.944$; RMSEA $=0.071,90 \%$-й доверительный интервал: 0.064-0.078) и статистически достоверно $\left(\triangle \chi^{2}=78.76\right.$, $\mathrm{df}=4, p<0.001)$ отличались от показателей модели с независимым вкладом трех базовых потребностей, что свидетельствует о необходимости учета различий между ними. Полученные результаты подтверждают исследования, показывающие, что различные базовые потребности могут быть связаны с различными результирующими переменными (Brien et al., 2012).

Описательная статистика и гендерные различия. Анализ гендерных различий в показателях удовлетворенности базовых психологических потребностей проводился с помощью t-критерия Стьюдента (таблица 3). Результаты свидетельствуют о несколько более высоком уровне удовлетворенности всех трех базовых потребностей у женщин, однако обнаруженные гендерные различия являются достаточно слабыми $(d<0.3)$. Показатели удовлетворенности базовых потребностей не продемонстрировали статистически достоверных связей со стажем работы и возрастом, что соответствует данным, полученным на западных выборках (Di Domenico, Fournier, 2014). Были получены лишь слабые, но статистически достоверные $(p<0.001)$ связи с должностным статусом, свидетельствующие о том, что более высокие должности предоставляют больше возможностей для удовлетворения базовых потребностей в компетентности $(\rho=0.18)$, автономии $(\rho=0.17)$ и связанности $(\rho=0.14)$. Данные, представленные в таблице 3, могут использоваться в качестве норм.

\section{Заключение}

Нами разработана Шкала удовлетворенности базовых потребностей на работе, теоретическим основанием которой выступает теория самодетерминации. Шкала обладает высоким уровнем надежности; показана ее конструктная валидность. Шкалу можно использовать в исследовательских целях для оценки степени удовлетворенности трех базовых психологических потребностей, а также в скринниговых целях.

Таблица 3

Описательная статистика шкал удовлетворенности базовых потребностей на работе $(\mathrm{N}=1183)$

\begin{tabular}{|c|c|c|c|c|c|c|c|c|}
\hline \multirow{2}{*}{$\begin{array}{c}\text { Базовые } \\
\text { психологические } \\
\text { потребности }\end{array}$} & \multicolumn{2}{|c|}{ Вся выборка } & \multicolumn{2}{|c|}{$\begin{array}{c}\text { Женщины } \\
\text { (N = 422) }\end{array}$} & \multicolumn{2}{|c|}{$\begin{array}{l}\text { Мужчины } \\
(\mathrm{N}=761)\end{array}$} & \multirow{2}{*}{$\mathrm{t}(1181)$} & \multirow{2}{*}{$\begin{array}{c}\text { Размер } \\
\text { эффекта } \\
\text { (d Коэна) }\end{array}$} \\
\hline & $M$ & $S D$ & $M$ & $S D$ & $M$ & $S D$ & & \\
\hline Автономия & 4.70 & 0.84 & 4.80 & 0.75 & 4.65 & 0.88 & -2.8 & 0.17 \\
\hline Компетентность & 5.06 & 0.81 & 5.18 & 0.77 & 4.99 & 0.82 & $-3.94 * * *$ & 0.24 \\
\hline Связанность & 5.11 & 0.86 & 5.23 & 0.82 & 5.05 & 0.88 & $-3.61 * * *$ & 0.22 \\
\hline
\end{tabular}

$* * p<0.01, * * * p<0.001$. 
Полученные данные свидетельствуют о важности удовлетворения базовых потребностей для поддержания продуктивного характера мотивации (прежде всего, внутренней мотивации) и субъективного благополучия у российских сотрудников. Результаты регрессионного анализа свидетельствуют о независимом вкладе каждой из базовых потребностей в показатели мотивации. Эти данные соответствуют как результатам западных (Ryan, Deci, 2000), так и российских исследований удовлетворенности базовых потребностей на материале учебной деятельности (Гордеева и др., 2013, 2014).

Удовлетворение БП на работе во многом определяется организационной средой (Шауфели и др., 2015), что свидетельствует о ценности разработки интервенций, направленных на их поддержку. Например, для поддержки потребности в автономии рекомендуется предоставлять работнику возможность проявлять инициативу, давать ему выбор внутри четкой зоны ответственности, обеспечивать его обратной связью неконтролирующего характера, позволяя сотруднику высказаться и почувствовать себя источником собственной деятельности, ее субъектом, а не объектом. Также актуально применение следующих стратегий: использовать меньше контролирующего языка («должен», «обязан»), команд, требований подчиниться, избегать управления с помощью «кнутов и пряников», системы компенсации труда, когда сотрудники эксплицитно конкурируют друг с другом, угроз наказаний и манипуляций с помощью разного рода материальных наград, чаще вести честные дискус- сии и диалоги, совместно решать проблемы, возникающие на работе, совместно вырабатывать правила и решения, использовать метод рефлексивного слушания, акцентирования самостоятельного выбора рабочих задач, способов их решения и пр., проявлять внимание к формам стимулирования мотивации, минимизировать акцент на компенсации труда и разного рода выплатах как стратегии мотивирования персонала.

С целью поддержки потребности сотрудников в компетентности необходимо, чтобы рабочие задачи соответствовали уровню способностей, умений и потенциала сотрудника, публично и открыто признавались их заслуги, предоставлялась обратная связь информативного, конструктивного характера, дающая возможность почувствовать себя эффективным деятелем, а также предоставлялись возможности для развития потенциала и талантов сотрудников. Наконец, для поддержки потребности в связанности с другими людьми важно поддерживать оптимальный социально-психологический климат, где работники чувствуют взаимное принятие и поддержку. Это может быть связано с нобходимостью отказа от систем ранжирования и разного рода рейтингования сотрудников с использованием кривых нормального распределения, разного рода соревнований, конкуренции и контроля, введения различных кооперативных форм взаимодействия, сотрудничества, проведения совместного досуга (например, совместные праздники).

Эти принципы не всегда легко внедрить в силу множества мифов о 
трудовой мотивации, существующих в современных организациях, но, как показывают современные психологические исследования и практические внедрения во множестве западных компаний (например, таких как «Xerox» и др.), их использование дает ощутимый прирост во внутренней и автономной мотивации сотрудников, способствуя снижению амотивации, росту креативности, поддержанию их психологического благополучия, снижению риска выгорания и росту доверия руководству.

\section{Литература}

Гордеева, Т. О., Сычев, О. А., Осин, Е. Н. (2013). Внутренняя и внешняя учебная мотивация студентов: источники и следствия для психологического благополучия. Вопросы психологии, 1 , $35-45$.

Гордеева, Т. О., Сычев, О. А., Осин, Е. Н. (2014). Опросник «Шкалы академической мотивации». Психологический журнал, 35(4), 96-107.

Осин, Е. Н. (2012). Измерение позитивных и негативных эмоций: разработка русскоязычного аналога методики PANAS. Психология. Журнал Высшей школы экономики, 9(4), 91-110.

Осин, Е. Н., Иванова, Т. Ю., Гордеева, Т. О. (2013). Автономная и контролируемая профессиональная мотивация как предикторы субъективного благополучия у сотрудников российских организаций. Организащионная психология, 3(1), 8-29. URL: http://orgpsyjournal.hse.ru/ data/2013/11/21/1337329670/OrgPsy_2013-1_Osin\%20et\%20al._8-29.pdf

Осин, Е. Н., Леонтьев, Д. А. (2008). Апробация русскоязычных версий двух шкал экспресс-оценки субъективного благополучия. Материаль ІІІ Всероссийского социологического конгресса. М.: Институт социологии РАН; Российское общество социологов.

Шауфели, В., Дийкстра, П., Иванова, Т. (2015). Увлеченность работой: Как научиться любить свою работу и получать от нее удовольствие. М.: Когито-Центр.

Baard, P. P., Deci, E. L., \& Ryan, R. M. (2004). Intrinsic need satisfaction: A motivational basis of performance and well-being in two work settings. Journal of Applied Social Psychology, 34, 2045-2068.

Brien, M., Forest, J., Mageau, G. A., Boudrias, J. S., Desrumaux, P., Brunet, L., \& Morin, E. (2012). The basic psychological needs at work scale: Measurement invariance between Canada and France. Applied Psychology: Health and Well-Being, 4(2), 167-187.

Brown, T. A. (2006). Confirmatory factor analysis for applied research. New York: The Guilford Press.

Chen, F. F., Hayes, A., Carver, C. S., Laurenceau, J.-P., \& Zhang, Z. (2012). Modeling general and specific variance in multifaceted constructs: A comparison of the bifactor model to other approaches. Journal of Personality, 80(1), 219-251.

Deci, E. L., \& Ryan, R. M. (2000). The "what" and "why" of goal pursuits: Human needs and the selfdetermination of behavior. Psychological Inquiry, 11, 227-268.

Deci, E. L., Ryan, R. M., Gagné, M., Leone, D. R., Usunov, J., \& Kornazheva, B. P. (2001). Need satisfaction, motivation, and well-being in the work organizations of a former Eastern Bloc country. Personality and Social Psychology Bulletin, 27, 930-942.

Di Domenico, S., \& Fournier, M. (2014). Socioeconomic status, income inequality, and health complaints: A basic psychological needs perspective. Social Indicators Research, 119, 1679-1697. 
Kasser, V. M., \& Ryan, R. M. (1999). The relation of psychological needs for autonomy and relatedness to health, vitality, well-being and mortality in a nursing home. Journal of Applied Social Psychology, 29, 935-954.

Lynch, M. F., Plant, R. W., \& Ryan, R. M. (2005). Psychological needs and threat to safety: Implications for staff and patients in a psychiatric hospital for youth. Professional Psychology, 36, 415-425.

Milyavskaya, M., Gingras, I., Mageau, G., Koestner, R., Gagnon, H., Fang, J., \& Boiche, J. (2009). Balance across contexts: The importance of balanced need satisfaction across various life domains in adolescence. Personality and Social Psychology Bulletin, 35(8), 1031-1045.

Reis, H. T., Sheldon, K. M., Gable, S. L., Roscoe, J., \& Ryan, R. M. (2000). Daily well-being: The role of autonomy, competence, and relatedness. Personality and Social Psychology Bulletin, 26, 419-435.

Ryan, R. M. (1995). Psychological needs and the facilitation of integrative processes. Journal of Personality, 63, 397-427.

Ryan, R. M., \& Deci, E. L. (2000). The darker and brighter sides of human existence: Basic psychological needs as a unifying concept. Psychological Inquiry, 11, 319-338.

Sheldon, K. M., Elliot, A. J., Kim, Y., \& Kasser, T. (2001). What is satisfying about satisfying events? Testing 10 candidate psychological needs. Journal of Personality and Social Psychology, 89, 325-339.

Sheldon, K. M., \& Hilpert, J. C. (2012). The balanced measure of psychological needs (BMPN) scale: An alternative domain general measure of need satisfaction. Motivation and Emotion, 36, 439-451.

Sheldon, K. M., \& Niemiec, C.P. (2006). It's not just the amount that counts: Balanced need satisfaction also affects well-being. Journal of Personality and Social Psychology, 91, 331-341.

Van den Broeck, A., Vansteenkiste, M., De Witte, H., \& Lens, W. (2008). Explaining the relationships between job characteristics, burnout and engagement: The role of basic psychological need satisfaction. Work and Stress, 22, 277-294.

Van den Broeck, A., Vansteenkiste, M., De Witte, H., Soenens, B., \& Lens, W. (2010). Capturing autonomy, competence, and relatedness at work: Construction and initial validation of the Work-related Basic Need Satisfaction Scale. Journal of Occupational and Organizational Psychology, 83, 981-1002.

\section{Текст методики}

Приложение

\section{«Шкала удовлетворенности базовых психологических потребностей на работе»}

Инструкция. Следующие утверждения описывают ваши ощущения от вашей работы за последний год (если вы работаете на этом месте меньше года, они относятся ко всему периоду вашей работы). Пожалуйста, оцените, насколько каждое из этих утверждений верно по отношению к вашим ощущениям на работе. Ваше руководство никогда не узнает о том, как вы ответили на эти вопросы. Пожалуйста, используйте следующую шкалу:

1

Совершенно

не верно
2

3

Отчасти

верно
5

6

Совершенно

верно 
1. Я чувствую, что могу участвовать в принятии решений о том, каким образом я должен(на) выполнять свою работу.

2. Люди, с которыми я работаю, мне по-настоящему нравятся.

3. Во время работы мне часто кажется, что мне не хватает знаний, опыта или способностей.

4. По мнению других, я хорошо справляюсь со своей работой.

5. На работе я чувствую, что на меня давят.

6. Мне хорошо удается ладить с людьми на работе.

7. Находясь на работе, я бо́льшую часть времени ни с кем не общаюсь.

8. Я чувствую, что могу свободно высказывать свои мысли и идеи относительно работы.

9. У меня теплые отношения с людьми, с которыми я работаю.

10. На работе мне удалось овладеть новыми интересными умениями (навыками).

11. Когда я на работе, я должен(на) делать то, что мне говорят.

12. В большинстве случаев после работы я чувствую, что успешно выполнил(а) ее.

13. Я чувствую, что на работе мое мнение и мои чувства принимают во внимание.

14. На работе у меня почти нет возможности показать, на что я действительно способен(на).

15. Люди на работе готовы помочь мне, поддержать меня.

16. На работе довольно мало людей, с которыми у меня установились близкие отношения.

17. Я чувствую, что на работе я могу вести себя естественно, быть самим(ой) собой.

18. Похоже, что я не слишком-то нравлюсь людям, с которыми работаю.

19. Во время работы я часто чувствую себя недостаточно способным(ой) к этому делу.

20. У меня довольно мало возможностей самому(ой) решать, как выполнять мою работу.

21. Люди на работе в основном дружелюбны со мной.

Обратные пункты (отмечены R) необходимо инвертировать. Показатели по шкалам рассчитываются как средние по следующим утверждениям:

Потребность в автономии:

Потребность в компетентности:

Потребность в связанности:
$1,5(\mathrm{R}), 8,11(\mathrm{R}), 13,17,20(\mathrm{R})$

$3(\mathrm{R}), 4,10,12,14(\mathrm{R})^{1}, 19(\mathrm{R})$

2, 6, 7(R), 9, 15, 16(R), 18(R), 21

${ }^{1}$ Утверждение 14 не использовалось при расчете общего показателя по шкале в рамках данной статьи; выше представлена доработанная формулировка пункта. 


\title{
Basic Psychological Need Satisfaction as a Source of Work Motivation and Subjective Well-being in Russian Employees
}

\section{Evgeny N. Osin}

Leading research fellow, International laboratory of positive psychology of personality and motivation HSE*; Associate professor, School of psychology HSE*, Ph.D.

E-mail: evgeny.n.osin@gmail.com

\section{Dmitry D. Suchkov}

Junior research fellow, International laboratory of positive psychology of personality and motivation HSE*; graduate student, School of psychology HSE*

E-mail: dsuchkov@hse.ru

\section{Tamara O. Gordeeva}

Associate professor, Department of psychology, Lomonosov Moscow State University**; Leading research fellow, International laboratory of positive psychology of personality and motivation HSE*, D.Sc.

E-mail: tamgordeeva@gmail.com

Tatiana Yu. Ivanova

Research fellow, International laboratory of positive psychology of personality and motivation HSE* E-mail: tatiana.y.ivanova@gmail.com

Address: * 20 Myasnitskaya str., Moscow, 101000, Russian Federation

** GSP-1, Leninskie Gory, Moscow, 119991, Russian Federation

\begin{abstract}
Satisfaction of basic psychological needs for autonomy, competence, and relatedness proposed in Self-Determination Theory (SDT) by E. Deci and R. Ryan is an important predictor of optimal functioning of individuals. The paper presents the results of validation of a Russian version of Basic Psychological Need Satisfaction at work scale developed by Deci et al. The study used an employee sample $(\mathrm{N}=1,183)$ from a large production plant in Russia. Structural equation modeling confirmed a good fit of a bifactor model including 3 factors of basic needs and 2 uncorrelated response bias factors corresponding to item direction. The scales demonstrate acceptable internal consistency (Cronbach's alpha in the $0.70-0.85$ range). To evaluate construct validity we used a work motivation questionnaire based on SDT and indicators of subjective well-being (life satisfaction and affect balance). The basic need satisfaction scales show weak to moderate positive association with life satisfaction and positive affect, as well as with intrinsic, integrated, identified, and introjected motivation scales. We also found negative associations of basic need satisfaction with negative affect, external motivation, and amotivation. Using structural equation modelling we show that autonomous and controlled work motivation can mediate the association between basic need satisfaction and subjective well-being. The findings are line with those of studies in other countries and with the predictions of SDT. We discuss the importance of development of interventions aimed to improve the basic psychological need satisfaction of employees and suggest guidelines for such interventions based in SDT.
\end{abstract}

Keywords: basic psychological needs, basic need satisfaction scales, self-determination theory, need for autonomy, need for competence, need for relatedness, work motivation, subjective well-being. 


\section{References}

Baard, P. P., Deci, E. L., \& Ryan, R. M. (2004). Intrinsic need satisfaction: A motivational basis of performance and well-being in two work settings. Journal of Applied Social Psychology, 34, 2045-2068.

Brien, M., Forest, J., Mageau, G. A., Boudrias, J. S., Desrumaux, P., Brunet, L., \& Morin, E. (2012). The basic psychological needs at work scale: Measurement invariance between Canada and France. Applied Psychology: Health and Well-Being, 4(2), 167-187.

Brown, T. A. (2006). Confirmatory factor analysis for applied research. New York: The Guilford Press.

Chen, F. F., Hayes, A., Carver, C. S., Laurenceau, J.-P., \& Zhang, Z. (2012). Modeling general and specific variance in multifaceted constructs: A comparison of the bifactor model to other approaches. Journal of Personality, 80(1), 219-251.

Deci, E. L., \& Ryan, R. M. (2000). The "what" and "why" of goal pursuits: Human needs and the selfdetermination of behavior. Psychological Inquiry, 11, 227-268.

Deci, E. L., Ryan, R. M., Gagné, M., Leone, D. R., Usunov, J., \& Kornazheva, B. P. (2001). Need satisfaction, motivation, and well-being in the work organizations of a former Eastern Bloc country. Personality and Social Psychology Bulletin, 27, 930-942.

Di Domenico, S., \& Fournier, M. (2014). Socioeconomic status, income inequality, and health complaints: A basic psychological needs perspective. Social Indicators Research, 119, 1679-1697.

Gordeyeva, T. O., Sychyov, O. A., \& Osin, E. I. (2013). Inner and outer motivation in students: Its sources and influence on psychological well-being. Voprosy Psikhologii, 1, 35-45.

Gordeeva, T. O., Sychev, O. A., \& Osin, E. N. (2014). "Academic Motivation Scales" questionnaire. Psikhologicheskii Zhurnal, 35(4), 96-107.

Kasser, V. M., \& Ryan, R. M. (1999). The relation of psychological needs for autonomy and relatedness to health, vitality, well-being and mortality in a nursing home. Journal of Applied Social Psychology, 29, 935-954.

Lynch, M. F., Plant, R. W., \& Ryan, R. M. (2005). Psychological needs and threat to safety: Implications for staff and patients in a psychiatric hospital for youth. Professional Psychology, 36, 415-425.

Milyavskaya, M., Gingras, I., Mageau, G., Koestner, R., Gagnon, H., Fang, J., \& Boiche, J. (2009). Balance across contexts: The importance of balanced need satisfaction across various life domains in adolescence. Personality and Social Psychology Bulletin, 35(8), 1031-1045.

Osin, E. N. (2012). Measuring positive and negative affect: Development of a Russian-language analogue of PANAS. Psychology. Journal of Higher School of Economics, 9(4), 91-110.

Osin, E. N., Ivanova, T. Y., \& Gordeeva, T. O. (2013). Autonomous and controlled professional motivation predict subjective well-being in Russian employees. Organizational Psychology - Russia (e-journal), 3(1), 8-29. Retrieved from http://orgpsyjournal.hse.ru/data/2013/11/21/1337329670/OrgPsy_20131_Osin\%20et\%20al._8-29.pdf

Osin, E. N., \& Leontiev, D. A. (2008). Aprobatsiya russkoyazychnykh versii dvukh shkal ekspressotsenki sub"ektivnogo blagopoluchiya [Aprobation of Russian versions of express-evaluation scales of subjective well-being]. Materialy III Vserossiiskogo Sotsiologicheskogo Kongressa [Proceedings of the III All-Russian Sociological Congress]. Moscow: Institute of Sociology of the Russian Academy of Sciences / Russian Society of Sociologists (ISBN 978-6-89697-157-3).

Reis, H. T., Sheldon, K. M., Gable, S. L., Roscoe, J., \& Ryan, R. M. (2000). Daily well-being: The role of autonomy, competence, and relatedness. Personality and Social Psychology Bulletin, 26, 419-435. 
Ryan, R. M. (1995). Psychological needs and the facilitation of integrative processes. Journal of Personality, 63, 397-427.

Ryan, R. M., \& Deci, E. L. (2000). The darker and brighter sides of human existence: Basic psychological needs as a unifying concept. Psychological Inquiry, 11, 319-338.

Schaufeli, W., Dijkstra, P., \& Ivanova, T. (2015). Uvlechennost' rabotoi: Kak nauchit'sya lyubit' svoyu rabotu i poluchat' ot nee udovol'stvie [Dedication to work: How to learn to love your work and enjoy it]. Moscow: Kogito-tsentr.

Sheldon, K. M., Elliot, A. J., Kim, Y., \& Kasser, T. (2001). What is satisfying about satisfying events? Testing 10 candidate psychological needs. Journal of Personality and Social Psychology, 89, 325-339.

Sheldon, K. M., \& Hilpert, J. C. (2012). The balanced measure of psychological needs (BMPN) scale: An alternative domain general measure of need satisfaction. Motivation and Emotion, 36, 439-451.

Sheldon, K. M., \& Niemiec, C.P. (2006). It's not just the amount that counts: Balanced need satisfaction also affects well-being. Journal of Personality and Social Psychology, 91, 331-341.

Van den Broeck, A., Vansteenkiste, M., De Witte, H., \& Lens, W. (2008). Explaining the relationships between job characteristics, burnout and engagement: The role of basic psychological need satisfaction. Work and Stress, 22, 277-294.

Van den Broeck, A., Vansteenkiste, M., De Witte, H., Soenens, B., \& Lens, W. (2010). Capturing autonomy, competence, and relatedness at work: Construction and initial validation of the Work-related Basic Need Satisfaction Scale. Journal of Occupational and Organizational Psychology. 83, 981-1002. 